\title{
Propuesta para la integración de las normas ISO 9001:2008 e ISO 14001:2004 en los procesos misionales de la Dirección Administrativa y Financiera de la Policía Nacional ${ }^{*}$
}

Proposal for integration of standards ISO 9001:2008 and ISO 14001:2004 in the missionary processes of the Administrative and Financial Direction of the National Police

Proposta para a inteğração das normas ISO 9001: 2008 e ISO 14001:2004 nos processos fundamentais da Diretoria Financeira e Administrativa da Polícia Nacional 


\section{RESUMEN}

El presente artículo pretende dar a conocer las actividades que se deben desarrollar para integrar el Sistema de Gestión de la Calidad y el Sistema de Gestión Ambiental en los procesos misionales de la Dirección Administrativa y Financiera de la Policía Nacional (DIRAF). Lo anterior a partir de la investigación y revisión documental realizada con el propósito de conocer la DIRAF y los diferentes métodos planteados por algunos autores y organizaciones para hacer la integración de los sistemas de gestión, así como la identificación de matrices 0 instrumentos que permitan determinar y aplicar una herramienta de medición para efectuar el análisis de contexto y conocer el grado de madurez en que se encuentra la implementación de los sistemas de gestión objeto de integración en la Dirección Administrativa y Financiera. Por último se indican los impactos positivos de tipo productivo, ambiental, económico y social, al realizar la integración de los sistemas en mención.

Palabras clave: contexto, gestión integral, integración, madurez, sistemas integrados, métodos de integración.

\section{ABSTRACT}

This article aims to present the activities must be developed to integrate the Quality Management System and Environment Management System in the missionary processes of the Administrative and Financial Direction of the National Police (DIRAF). This is based on the investigation and documentary review done with the purpose of to know the DIRAF and the different methods proposed by some authors and organizations to do the integration of the systems management, as well as the identification of matrices or instruments that allow to determinate and to apply a measuring tool to do the context analysis and to know the degree of maturity of the implementation of the management systems that are object the integration in the Administrative and Financial Direction. Finally, shows the positive impacts in productive, environmental, economic and social topics, when the integration of the mentioned systems is carried out.

Keywords: context, integral management, integration, maturity, integrated systems, methods of integration.

\section{RESUMO}

Este artigo procura destacar as atividades que devem ser desenvolvidas para integrar o Sistema de Gestão de Qualidade e o Sistema de Gestão Ambiental nos processos fundamentais da Direção Administrativa e Financeira da Polícia Nacional (DIRAF). A partir da revisão de documentos e pesquisa realizada com 0 objetivo de conhecer a DIRAF e os diferentes métodos propostos por alguns autores e organizações para fazer a integração dos sistemas de gestão, bem como a identificação de matrizes ou dispositivos que permitam determinar e aplicar uma ferramenta de medida para efetuar a análise do contexto e conhecer 0 nível de desenvolvimento em que se encontra a implementação dos sistemas de gestão sujeitos a integração na Direção Administrativa e Financeira. Finalmente são mostrados os impactos positivos no âmbito produtivo, ambiental, econômico e social, ao realizar a integração dos sistemas mencionados.

Palavras-chave: contexto, gestão de integração, integração, desenvolvimento, sistemas integrados, métodos de integração.

\section{INTRODUCCIÓN}

La Dirección Administrativa y Financiera de la Policía Nacional, desde el año 2009, logró la certificación del 
Sistema de Gestión de la Calidad bajo los requisitos de la norma ISO 9001:2008, así mismo, en el año 2013 obtuvo la certificación del Sistema de Gestión Ambiental bajo los requisitos de la NTC ISO 14001:2004, de manera independiente otorgadas por el ICONTEC, las cuales se han mantenido conformes. Esta Dirección es la responsable de liderar a nivel de la Dirección General de la Policía Nacional, la implementación, mantenimiento y mejora del Sistema de Gestión Ambiental, con el apoyo de la Oficina de Planeación la cual establece los lineamientos a seguir en el sistema.

Dado que los sistemas de gestión fueron implementados de manera independiente y en años diferentes, lo que equivale a que la administración de los mismos se hace de la misma manera, es necesario encontrar los puntos de convergencia para lograr mayor eficacia y eficiencia en la ejecución y resultados de estos dos sistemas.

Teniendo en cuenta las necesidades de integración, se desarrolló el trabajo para establecer la revisión inicial de los Sistemas de Gestión de la Calidad y de Gestión Ambiental en los procesos misionales de la Dirección Administrativa y Financiera de la Policía Nacional, se aplicaron las matrices de verificación de requisitos y esquemas para un análisis de contexto, con el propósito de identificar el método de integración más adecuado y generar una propuesta para realizar la integración de los sistemas de gestión anteriormente mencionados, los cuales ya están implementados en esta Dirección.

El diseño del sistema integrado de gestión, permite una mejor captación por parte de los funcionarios sobre los requisitos ambientales y de calidad de los diferentes procesos, así como la optimización de recursos a nivel económico, de gestión, de mejoramiento continuo de la calidad, de tiempo de los procesos y mejora en las condiciones ambientales de la Dirección Administrativa y Financiera y en el edificio en general, en donde esta Dirección tiene su sede.
Uno de los beneficios de realizar la propuesta de integración de las normas ISO 9001:2008 e ISO 14001:2009, es que esto puede constituirse en una ayuda para que otras direcciones de la Policía Nacional de Colombia realicen la integración de los sistemas.

El objetivo general del trabajo, es elaborar una propuesta de integración de las normas ISO 9001:2008 e ISO 14001:2004 en los procesos misionales de la Dirección Administrativa y Financiera de la Policía Nacional.

Para cumplir con este objetivo, se determinaron los siguientes objetivos específicos:

- Hacer una revisión inicial de los Sistemas de Gestión de la Calidad y de Gestión Ambiental, para determinar el nivel de madurez para la integración en los procesos misionales de la Dirección Administrativa y Financiera de la Policía Nacional.

- Identificar cuál es el método más apropiado para realizar la integración de la ISO 9001 y la ISO 14001 para los procesos misionales de la Dirección Administrativa y Financiera de la Policía Nacional.

- Utilizar una herramienta que permita establecer las actividades a realizar para la integración de los sistemas.

La hipótesis que se pretende verificar es que la falta de un sistema de gestión integrado que contemple el Sistema de Gestión de la Calidad y el Sistema de Gestión Ambiental, en los procesos misionales de la Dirección Administrativa y Financiera, provoca duplicidad de funciones y documentos, auditorías separadas y menor eficiencia en el desarrollo de los mismos.

Como lo indican Abad y Sánchez (2012), el alcance de un sistema integrado de gestión se define como el conjunto de sistemas de gestión que lo conforman y depende de las necesidades y circunstancias de la organización. Para la década de los noventa, el alcance de la integración se limitaba únicamente a fusionar la 
documentación exigida por los sistemas de gestión de calidad y ambiental, buscando como objetivo reducir esfuerzos y costos.

Según López Carrizosa (2008), para lograr la integración adecuada de los sistemas de gestión, se debe tener un alto compromiso por parte de la Dirección y del personal que integra la institución, así mismo, se debe tener el conocimiento de los diferentes requisitos y un adecuado nivel de madurez del sistema.

De acuerdo a lo indicado por Santana (2010), autores como Inin, Isaac y Vásquez, "se basan para el diseño e implantación de un Sistema de Gestión Integrado, en el ciclo Deming, ya que se planifica todo el proceso de diseño, luego se ejecuta el mismo y se implanta, posteriormente se revisa y se mide el sistema y se toman acciones de mejoras para eliminar y reducir las deficiencias" (p. 2).

Para Carmona (2008), La integración sobre la base de los procesos se debe gestionar para obtener productos que cumplan tanto los requisitos del cliente, como los ambientales. Es por esta razón que con la ayuda de un panel de profesionales (participación de profesionales expertos con experiencia en las materias analizadas), da a conocer de una manera más práctica y analizando la norma UNE 66177-2005 "Guía para la integración de los sistemas de gestión”, la forma de cómo integrar los sistemas de gestión basándose en la gestión por procesos, explicando los tres métodos de integración relacionados en la misma: método básico, método avanzado y método experto.

\section{METODOLOGÍA}

El trabajo se fundamentó en el paradigma pragmático, toda vez que permite una investigación de tipo mixta, con un análisis cualitativo y cuantitativo y tiene varias posibilidades para construir este análisis.
Según el propósito o finalidad de la investigación, según Vargas (2009), esta se caracteriza por buscar la aplicación o utilización de los conocimientos establecidos desde la teoría formal y los que se generen después de realizada y practicada la investigación. Lo anterior se observa al analizar los métodos de integración de los sistemas y realizar la propuesta de integración para la Dirección Administrativa y Financiera.

De acuerdo a Hernández Sampieri (2014), el método utilizado fue de tipo mixto secuencial, ya que el objetivo de este trabajo era realizar la integración de dos normas ya certificadas en la Dirección Administrativa y Financiera de la Policía Nacional. En la primera etapa se desarrolló un análisis inicial cualitativo de los métodos de integración de la institución (revisión inicial), los datos se ampliaron y profundizaron con una segunda etapa de tipo cuantitativo donde se realizó el análisis del cumplimiento de requisitos de cada norma para lograr su integración y el estudio del nivel de madurez de la misma; en cada una de las etapas se realizó una exhaustiva revisión bibliográfica y recolección de datos, a partir de ello se hizo una exploración e interpretación de resultados.

Para calcular el nivel de madurez de los sistemas de gestión a integrar en la Dirección Administrativa y Financiera, se ajustó la matriz de análisis de contexto diseñada por el panel de profesionales y expertos del documento de Carmona (2008), y se aplicó a cada uno de los responsables de los procesos misionales de la DIRAF en forma personal y a través de correo electrónico.

\section{RESULTADOS Y DISCUSIÓN}

Para dar inicio al proceso de integración de los sistemas de gestión, se realizó en primera instancia un análisis de la institución, observando que la misma fue creada mediante el Decreto $\mathrm{N}^{\circ} 1000$ expedido por el Gobierno Nacional, el 5 de noviembre de 1891; den- 
tro del mapa de procesos de primer nivel de la Policía Nacional, la Dirección Administrativa y Financiera se encuentra como un elemento de soporte con las áreas de administración de recursos financieros y logística y abastecimiento, y esta Dirección, cuenta con un mapa de ruta el cual tiene procedimientos gerenciales, de soporte, de evaluación y mejora, y los procesos misionales, estos últimos, objeto de estudio para la integración de las normas ISO 9001:2008 e ISO 14001:2004.

Con el propósito de conocer el estado actual de la implementación del Sistema de Gestión de la Calidad (ISO 9001:2008) y del Sistema de Gestión Ambiental (ISO 14001:2004) en la Dirección Administrativa y Financiera (DIRAF), se desarrolló el proceso de verificación de cumplimiento de requisitos basado en las normas mencionadas anteriormente; con un componente cuantitativo y cualitativo, por medio de matrices de "Verificación y cumplimiento de requisitos norma ISO 9001:2008 en la DIRAF" y "Verificación y cumplimiento de requisitos norma ISO 14001:2004 en la DIRAF", en donde se relacionan los documentos a través de los que se da cumplimiento a cada uno de estos requisitos.

Posteriormente a la verificación de los requisitos establecidos por las normas, se evidenció que la Dirección Administrativa y Financiera, cumple con el $100 \%$ de los mismos, lo cual permitió indicar que es una instancia apta para realizar un proceso de integración basado en las normas mencionadas.

Una vez verificado el cumplimiento de los requisitos de los dos sistemas objeto de integración, se procedió a determinar cuáles de ellos en la actualidad se encuentran integrados en la Dirección Administrativa y Financiera, por cuanto ya tienen un procedimiento establecido para el desarrollo del mismo. Se evidenció que el $42 \%$ de los requisitos comunes de la norma ISO 9001:2008 (equivalentes a 14 de 33), se encuentran integrados con el $37 \%$ de los requisitos comunes de la norma ISO 14001:2004 (equivalentes a 7 de 19).

Teniendo en cuenta lo anterior, se observa que el 58 $\%$ de los requisitos comunes de la ISO 9001:2008 y el $63 \%$ de la ISO 14001:2004, faltan por ser integrados.

De otra parte y teniendo en cuenta que la norma UNE 66177:2005 (AENOR, 2005) indica que, "como herramienta de gestión para el análisis de la situación de partida, puede ser interesante que la organización realice un análisis DAFO (análisis de las debilidades, amenazas, fortalezas y oportunidades) de su situación u otra técnica similar" (p. 9), a continuación se presenta el DAFO de la DIRAF.

Tabla 1. Análisis DAFO - Dirección Administrativa y Financiera.

\begin{tabular}{|c|c|}
\hline Fortalezas & Debilidades \\
\hline $\begin{array}{l}\text { La Dirección Administrativa y Financiera se encuentra certificada con la NTC- } \\
\text { GP 1000:2009, ISO 9001:2008, ISO 14001:2004 e ISO IEC 27001:2013. } \\
\text { Su enfoque es basado en procesos. } \\
\text { La Dirección General de la Policía se encuentra certificada con la norma ISO } \\
\text { 14001:2004, comprometiéndose con el cuidado del medio ambiente. } \\
\text { La alta dirección es comprometida con la mejora continua de los sistemas de } \\
\text { gestión normalizados. } \\
\text { Cuenta con una plataforma virtual como lo es la Polired, la herramienta Suite } \\
\text { Vision Empresarial SVE, aplicativos como el SIIF, SIBIN, SIGEA, SIFAC, entre } \\
\text { otros, permitiendo una excelente comunicación con las partes interesadas y así } \\
\text { tener un mejor control de los productos y servicios ofrecidos. } \\
\text { Cuenta con personal comprometido en el ejercicio de sus funciones. } \\
\text { Realiza seguimiento constante a los indicadores de gestión con el propósito de } \\
\text { tomar medidas y acciones pertinentes en caso de desviación. } \\
\text { Estructura organizacional bien definida. }\end{array}$ & $\begin{array}{l}\text { Los sistemas de gestión certificados en la DIRAF no se en- } \\
\text { cuentran integrados, duplicando documentos y funciones. } \\
\text { Los procesos y procedimientos establecidos por la DIRAF, no } \\
\text { establecen aspectos ambientales. } \\
\text { Un porcentaje bajo de funcionarios se resiste al cambio. } \\
\text { Las auditorías a los sistemas de gestión se realizan de forma } \\
\text { separada. } \\
\text { Hay funcionarios que no cumplen con el perfil del cargo } \\
\text { ejercido. } \\
\text { Los funcionarios no tienen claro conocimiento sobre los re- } \\
\text { quisitos de la norma ISO } 14001 \text {. } \\
\text { Falta mayor compromiso hacia el cumplimiento de la política } \\
\text { de gestión ambiental por parte de los funcionarios. }\end{array}$ \\
\hline
\end{tabular}




\begin{tabular}{|c|c|}
\hline Oportunidades & Amenazas \\
\hline $\begin{array}{l}\text { Convenios de apoyo con entidades educativas a nivel nacional en grado profe- } \\
\text { sional y técnico. } \\
\text { Las políticas del Estado frente al cuidado y preservación del medio ambiente. } \\
\text { Alianzas con las Policías del mundo para referenciar y ser referenciados. } \\
\text { Incorporación de personal profesional y especializado en diferentes áreas. }\end{array}$ & $\begin{array}{l}\text { No existe una norma que certifique los sistemas de gestión de forma } \\
\text { integrada. } \\
\text { Los lineamientos institucionales dependen de políticas del Estado. } \\
\text { Cambios normativos en las diferentes áreas, ocasionando retrocesos } \\
\text { en la gestión. } \\
\text { Altos costos para la adquisición de tecnologías limpias y con criterios } \\
\text { sostenibles. }\end{array}$ \\
\hline
\end{tabular}

Fuente: el autor.

En la literatura actual se ha encontrado que varios autores han desarrollado propuestas para la integración de sistemas en varios niveles, así:

Tabla 2. Aspectos clave de la integración de sistemas de gestión.

\begin{tabular}{|c|c|c|c|}
\hline Autores & $\begin{array}{l}\mathrm{N}^{\circ} \text { de } \\
\text { niveles }\end{array}$ & $\begin{array}{l}\text { Nombre de los } \\
\text { niveles }\end{array}$ & Descripción \\
\hline \multirow{3}{*}{ Seggezzi (1997) } & \multirow{3}{*}{3} & 1. Suma & $\begin{array}{l}\text { Los sistemas se mantienen separados, así como su documentación. Se desarrollan listas } \\
\text { cruzadas para comparar el contenido. }\end{array}$ \\
\hline & & 2. Fusión & $\begin{array}{l}\text { Se integra completamente el nivel operacional (funciones únicas y descritas para los } \\
\text { empleados), existe un único manual de gestión. } \\
\text { Se mantienen visibles capítulos específicos de los sistemas. }\end{array}$ \\
\hline & & 3. Integración & Un único sistema global que no permite que los sistemas se vean por separado. \\
\hline \multirow{4}{*}{$\begin{array}{l}\text { Wilkinson y } \\
\text { Dale (1999) }\end{array}$} & \multirow{4}{*}{4} & Primer nivel & $\begin{array}{l}\text { Se alcanza cuando un sistema individual se integra en todas y cada una de las actividades } \\
\text { de la organización. }\end{array}$ \\
\hline & & Segundo nivel & Se combinan los sistemas de gestión a través de tablas de correspondencia. \\
\hline & & Tercer nivel & $\begin{array}{l}\text { Aparición de sistemas satélites que gestionan aspectos comunes de los sistemas, como } \\
\text { por ejemplo: formación. }\end{array}$ \\
\hline & & Cuarto nivel & $\begin{array}{l}\text { Se fusionan los sistemas certificados o no, en un solo sistema global alineando sus polí- } \\
\text { ticas y objetivos. }\end{array}$ \\
\hline \multirow{2}{*}{$\begin{array}{l}\text { Block y Marash } \\
\qquad(2000)\end{array}$} & \multirow{2}{*}{2} & 1. Parcial & $\begin{array}{l}\text { Los procedimientos se realizan en forma separada, existen manuales independientes } \\
\text { y solo cuenta con documentos compartidos en los sistemas (control de documentos). } \\
\text { Su estructura organizativa es descentralizada y con un estilo de gestión autocrático. }\end{array}$ \\
\hline & & 2. Total & $\begin{array}{l}\text { Existe un único manual, los sistemas pierden su identidad, las auditorías y la revisión } \\
\text { por la Dirección es única. } \\
\text { Su estructura organizativa es centralizada y con un estilo de gestión participativo. }\end{array}$ \\
\hline \multirow{3}{*}{$\begin{array}{l}\text { Ferguson et al. } \\
\qquad(2002)\end{array}$} & \multirow{3}{*}{3} & 1.Alineamiento & Integración documental. \\
\hline & & 2.Combinación & $\begin{array}{l}\text { Se integra la documentación y los procedimientos de: control documental, registro de } \\
\text { datos, acciones correctivas y preventivas, formación y auditoría interna. }\end{array}$ \\
\hline & & 3.Integración & $\begin{array}{l}\text { Se realiza la integración de todo lo anterior, las políticas, los planes y objetivos; y los pro- } \\
\text { cedimientos de emergencia, sistema de evaluación y revisión de resultados y autoridad } \\
\text { y dirección. }\end{array}$ \\
\hline
\end{tabular}




\begin{tabular}{|c|c|c|c|}
\hline Autores & $\begin{array}{l}\mathrm{N}^{\circ} \text { de } \\
\text { niveles }\end{array}$ & Nombre de los niveles & Descripción \\
\hline \multirow{3}{*}{$\operatorname{AENOR~(2005)~}$} & \multirow{3}{*}{3} & 1. Básico & $\begin{array}{l}\text { Integración documental: políticas, manual de gestión, identificación y } \\
\text { acceso a requisitos legales. }\end{array}$ \\
\hline & & 2. Avanzado & $\begin{array}{l}\text { Requiere de experiencia en la gestión por procesos. Se integra la revisión } \\
\text { por la Dirección, comunicación, información y participación y procesos } \\
\text { productivos. Mapa de procesos integrado. }\end{array}$ \\
\hline & & 3.Experto & $\begin{array}{l}\text { La integración va más allá de los sistemas de gestión de la calidad, am- } \\
\text { biental y seguridad y salud laboral. }\end{array}$ \\
\hline \multirow{3}{*}{$\begin{array}{l}\text { Jorgensen et al. } \\
\qquad(2006)\end{array}$} & \multirow{3}{*}{3} & 1. Correspondencia & $\begin{array}{l}\text { Sistemas separados y su relación es por tablas de correspondencias, existe } \\
\text { un único manual de gestión. }\end{array}$ \\
\hline & & 2. Coordinación & $\begin{array}{l}\text { Se ejecutan integradamente los procesos genéricos: política, planifica- } \\
\text { ción, implantación y acciones preventivas y correctivas, revisión por la } \\
\text { Dirección. }\end{array}$ \\
\hline & & 3. Estratégico & $\begin{array}{l}\text { Implica la implantación de una cultura organizativa respecto al sistema } \\
\text { integrado, compromiso de la alta dirección, motivación y participación de } \\
\text { los empleados. }\end{array}$ \\
\hline
\end{tabular}

Fuente: adaptado de Abad y Sánchez.

De acuerdo a la Asociación Española de Normalización y Certificación (AENOR, 2005), "Con el fin de definir la mejor manera para realizar la integración, se hace necesario conocer la institución desde el punto de vista tanto interno y su relación con el entorno, esto se denomina análisis de contexto" (p. 6).

La norma UNE 66177:2005, plantea cuatro variables a evaluar para desarrollar el análisis de contexto de una organización: madurez, complejidad, alcance y riesgo, siendo la primera la más importante de las cuatro, por cuanto esta se mide en cinco grados, de los cuales, si la entidad solo aprueba el primero, su integración se verá limitada al método básico. Los grados son los siguientes:

- Inicial (sin aproximación formal): las actividades y los procesos de la organización se realizan total o parcialmente, pero no se documentan de manera adecuada.

- Básico (aproximación reactiva): las actividades y los procesos se realizan totalmente y se documentan de manera adecuada. Existe además un seguimiento mínimo de su eficacia.
- $\quad$ Avanzado (aproximación del sistema de forma estable): se toman acciones derivadas del seguimiento y de la medición de datos, por lo que se produce una tendencia a la mejora en etapas tempranas a la mejora de esta.

- $\quad$ Experto (énfasis en la mejora continua): el proceso es eficaz y eficiente, a la vez que existe una tendencia mantenida a la mejora.

- $\quad$ Premio (desempeño de "mejor en su clase"): las actividades y procesos se realizan y revisan teniendo en cuenta lo que hacen los mejores en el sector y midiendo el nivel de satisfacción de las partes afectadas (Abad y Sánchez, 2012, p. 76).

Para realizar el análisis de contexto de la Dirección Administrativa y Financiera, se tomaron las matrices desarrolladas por el Centro Andaluz para la Excelencia en la Gestión, las cuales fueron diseñadas por un panel de profesionales que tenían conocimientos y experiencias en la gestión normalizada, quienes determinaron las variables necesarias para la medición de los parámetros de madurez, complejidad, alcance y riesgo, y validada por las organizaciones participantes. 
Este instrumento se aplicó a los responsables de los procesos misionales de la Dirección Administrativa y Financiera de la Policía Nacional (DIRAF) y al responsable de calidad de la Oficina de Planeación.

De acuerdo al instrumento aplicado, se obtuvo como resultado un nivel de madurez avanzado que, según la norma, se define así: "la actividad o proceso se realiza y revisa; se toman acciones derivadas del seguimiento y análisis de datos. Existe tendencia a la mejora en etapas tempranas del proceso" (AENOR, 2005, p. 12).

Se evidencia que la DIRAF cuenta con un direccionamiento estratégico basado en la gestión de la calidad, además de concentrar toda su gestión en el enfoque basado en procesos. Ahora bien, toda vez que el resultado del nivel de madurez fue avanzado, de acuerdo a lo establecido en la norma se deben evaluar los siguientes tres parámetros para establecer el método de integración más apropiado:

- $\quad$ Nivel de complejidad: en este aparte se deben tener en cuenta las necesidades y expectativas de todas las partes interesadas, considerando las estrategias de la propia organización. Para definir el nivel de complejidad de la institución, se toma como insumo el instrumento facilitado en la investigación realizada por Carmona (2008), el cual define una complejidad en tres niveles: alto, medio y bajo. El resultado para este parámetro en la DIRAF es el nivel medio.

- $\quad$ Nivel de riesgo: este punto se relaciona con el incumplimiento a requisitos legales o fallos asociados al proceso de integración, como los aspectos ambientales. Como en el anterior nivel, el instrumento utilizado en este paso es extraído de la investigación de Carmona, en donde define los niveles de riesgo; aplicado este instrumento, se determina que la DIRAF cuenta con un nivel de riesgo medio, esto se traduce en que se deben tener claros y se deben intervenir los riesgos directamente relacionados con la integración de los sistemas.
- $\quad$ Nivel de alcance: este paso se relaciona con la visión con la que cuenta la entidad a mediano y largo plazo, teniendo en cuenta sus partes interesadas, se basa en la extensión que se quiere tener con los sistemas de gestión. Como en los anteriores puntos, el instrumento utilizado en este paso es extraído de la investigación de Carmona. Una vez desarrollado el instrumento, se define que el nivel de alcance de la DIRAF se encuentra en un nivel medio, esto relacionado con la dispersión de las unidades en donde los sistemas de gestión deben implementarse.

Una vez realizado el análisis de contexto y obtenidos los resultados, se debe tener en cuenta la siguiente escala de calificación para evidenciar el nivel de madurez de la institución:

- Si el valor numérico del promedio es > 2.3: ALTO

- Si el valor numérico del promedio es < 1.4: BAJO

- Si el valor numérico del promedio está entre 1.4 y 2.3: MEDIO

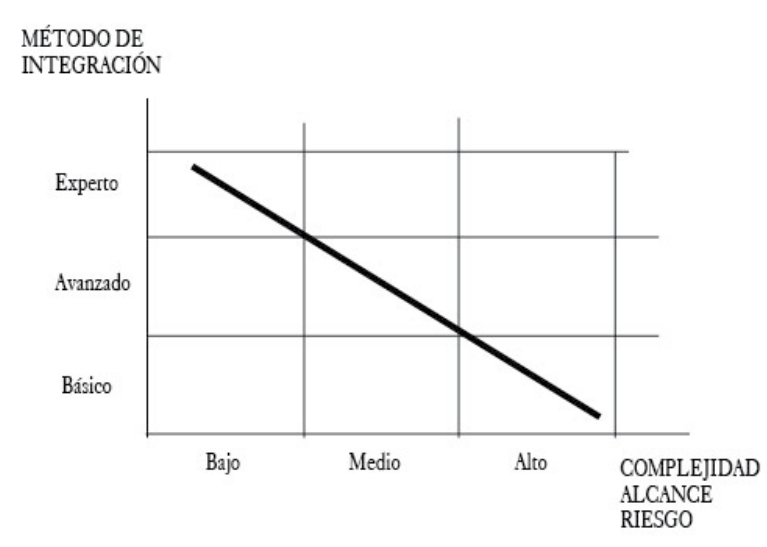

Fuente: norma UNE 66177:2005.

Figura 1. Nivel de madurez.

Este análisis evidenció que la Dirección Administrativa y Financiera se encuentra en el nivel medio por cuanto arrojó los siguientes resultados: 
Tabla 3. Calificación nivel de madurez.

\begin{tabular}{lc}
\multicolumn{1}{c}{ Parámetro } & Calificación \\
\hline Madurez & Avanzada \\
Complejidad & 1,75 \\
Alcance & 1,65 \\
Riesgo & 2,13
\end{tabular}

De acuerdo a la revisión inicial y al resultado del análisis de contexto de la Dirección Administrativa y Financiera, y con el propósito de que la propuesta de integración sea exitosa, se tuvo en cuenta el esquema de la matriz realizada por Gatell y Pardo (2014), la cual presenta la correspondencia entre los requisitos integrados y las normas a integrar, de tal forma que se pueden evidenciar los requisitos comunes, homologados y específicos de las mismas a partir del ciclo PHVA (planificar, hacer, verificar, actuar).

Así mismo, se establecieron unos lineamientos para la integración de los requisitos comunes de la norma ISO 9001:2008 con la norma ISO 14001:2004, teniendo en cuenta lo siguiente: descripción de requisitos generales, política integrada, enfoque al cliente, objetivos y programas, requisitos legales y otros requisitos, provisión de recursos, funciones, responsabilidades y autoridad, competencia, formación y toma de conciencia, comunicación, control de documentos, infraestructura, control operacional, control de equipos de seguimientos y evaluación.

\section{CONCLUSIONES}

$\mathrm{Al}$ desarrollar los objetivos establecidos para lograr la integración de los Sistemas de Gestión de la Calidad y de Gestión Ambiental, se pudo establecer que la Dirección Administrativa y Financiera se encuentra en un grado de madurez medio, lo que implica que la integración se puede obtener en un corto plazo.
Se evidencia que el grado de cumplimiento de los requisitos de los Sistemas de Gestión de la Calidad y de Gestión Ambiental es del $100 \%$, lo que ha permitido obtener y mantener las certificaciones otorgadas por el ICONTEC.

Con el grado de madurez que tiene el sistema, se deduce que ya existe una cultura organizacional por parte del personal que integra la institución policial, para trabajar con un enfoque basado en procesos y permite cumplir con los requisitos del Sistema de Gestión de la Calidad y del Sistema de Gestión Ambiental, garantizando continuidad en la prestación de servicios y cumplimiento de la normatividad legal.

Se pudieron identificar a través de la matriz de correspondencia los elementos comunes de los sistemas objeto de integración, esto facilita la integración de estas dos normas en un corto plazo.

La Dirección Administrativa y Financiera cuenta con personal capacitado en las normas ISO 9001 e ISO 14001, lo cual es una fortaleza para la creación del equipo de trabajo que puede integrar las mismas, dado el grado de conocimiento de los procesos que se ejecutan.

De acuerdo con el nivel de madurez que tiene la entidad, se establece que el método más apropiado para realizar la integración de los requisitos de las normas del Sistema de Gestión de la Calidad y del Sistema de Gestión Ambiental, es el método avanzado, de acuerdo con la metodología desarrollada por Miguel Ángel Carmona Calvo (2008).

Además de realizar una integración documental, se debe realizar un mapa de procesos que integre los sistemas de gestión y sus interrelaciones, definir en los procesos el responsable del mismo, indicadores, elementos de entrada y salida, formación, planificación, procesos relacionados con el cliente, mantenimiento, equipos de seguimiento y medición, revisión y mejora 
sistemática de los procesos teniendo en cuenta los requisitos de cada sistema.

Para lo anterior, se puede tener en cuenta la matriz para la integración de los Sistemas de Gestión ISO 9001:2008 e ISO 14001:2004, la cual indica las evidencias habituales de acuerdo con el ciclo PHVA, mediante la cual se hizo una correlación de los requisitos, estableciendo las acciones que se pueden llevar a cabo para lograr esta integración, lo que facilita a la institución la formulación de las acciones a llevar a cabo en un corto plazo.

Para la Dirección Administrativa y Financiera, dada su misionalidad y de acuerdo con los resultados obtenidos en el presente trabajo, es muy beneficiosa la integración de estos sistemas, ya que puede optimizar los recursos y disminuir los impactos ambientales, lo que podría ser realizado en un corto plazo, teniendo en cuenta la cultura institucional y los certificados que ya tiene frente a estos sistemas de gestión.

Los procedimientos establecidos por la DIRAF en los procesos misionales de logística y abastecimiento, administración de recursos financieros y adquisición de bienes y servicios, no incluyen el componente ambiental en las actividades descritas dentro del mismo.

La misionalidad de la Dirección Administrativa y Financiera es la de administrar los recursos logísticos y financieros de la Policía Nacional, razón por la cual es muy beneficioso a nivel de costos la integración de los requisitos de las dos normas, con lo cual puede esta Dirección ser más eficiente en la ejecución de los recursos financieros asignados.

Con la integración de los requisitos en los procesos de la DIRAF, se logran impactos de gran relevancia como son el cumplimiento de la responsabilidad social, contribuyendo a la protección del medio ambiente y de los recursos naturales, lo cual posiciona a la institución policial y mejora la percepción ciudadana.

Dados los impactos ambientales generados por los procesos, la integración garantiza que con la inclusión de los controles operacionales dentro de los procedimientos, se estandarice su aplicación por parte del personal que los ejecuta, logrando interiorización y cultura institucional.

El manejo de los recursos logísticos, implica que la institución disponga dentro de sus procesos actividades de control, que permitan garantizar la disposición final de los bienes sin que se afecte el medio ambiente y con la inclusión de los requisitos de carácter ambiental, se garantiza que los impactos ambientales no tengan un alto grado de afectación.

Teniendo en cuenta que se evidenció que la Dirección Administrativa y Financiera cumple al $100 \%$ con los requisitos de los dos sistemas (de Gestión Ambiental y de Gestión de la Calidad), se evidencia que la integración no se ha dado debido a que la implementación fue realizada de manera independiente en la institución policial de acuerdo a políticas institucionales y tiempos de certificación, de igual manera se realizaron las auditorías de manera independiente, sin que se hubiese desarrollado un estudio que permitiera demostrar el grado de cumplimiento en forma integral de los requisitos y su corresponsabilidad, lo que permitiría tomar una decisión al alto mando institucional sobre esta integración.

Se concluye que la Dirección Administrativa y Financiera a través de la implementación de un plan de acción, puede llevar a cabo la integración de estas dos normas sin que esto implique una inversión alta de recursos económicos, ya que el mayor impacto estará en la integración documental y su estandarización.

Se recomienda crear un grupo de trabajo con personal capacitado o que tenga un conocimiento previo de los 
Sistemas de Gestión de la Calidad y de Gestión Ambiental, para que se elabore el plan de trabajo que permita la integración de las dos normas que actualmente tiene certificadas la DIRAF.

\section{REFERENCIAS}

Abad Puente, J., \& Sánchez-Toledo Ledesma, A. (2012). Aspectos clave de la integración de sistemas de gestión. Madrid, España: AENOR Ediciones.

AENOR. (2005). Asociación Española de Normalización y Certificación. Sistemas de gestión. Guía para la integración de los sistemas de gestión. Norma UNE 66177. Madrid, España.

Carmona Calvo, M. Á. (2008). La integración de sistemas de gestión normalizados sobre la base de los procesos, Estudio a partir de un panel de expertos en el ámbito del Centro Andaluz para la Excelencia en la Gestión. Recuperado de: http://studylib.es/doc/5615118/
la-integraci\%C3\%B3n-de-sistemas-degesti\%C3\%B3n-normalizados-sobre-...

Gatell Sánchez, C., y Pardo Álvarez, J. M. (2014). Éxito de un sistema integrado. Madrid, España: AENOR Ediciones.

Hernández Sampieri, R., Fernández Collado, C., y Baptista Lucio, P. (2014). Metodología de la investigación. Sexta Edición. México D. F., México: McGraw-Hill.

López Carrizosa, F. J. (2008). El sistema de gestión integrado. Bogotá D. C., Colombia: ICONTEC.

Santana, K. (2010). Diseño e implantación del sistema integrado de gestión de la calidad, medio ambiente y seguridad y salud del trabajo (tesis de maestría). Instituto Superior Politécnico José Antonio Echeverría CUJAE, La Habana, Cuba.

Vargas Cordero, Z. R. (2009). La investigación aplicada: una forma de conocer las realidades con evidencia científica. Educación, 33(1). 